\title{
Prevalencia de estomatitis subprotésica, queilitis angular e hiperplasia fibrosa asociadas al uso de prótesis dental en una clínica universitaria de Medellín, 2017
}

The prevalence of sub-prosthetic stomatitis, angular cheilitis and fibrous hyperplasia associated with the use of dental prostheses in a university clinic in Medellín, 2017

The prevalence of sub-prosthetic stomatitis, angular cheilitis and fibrous hyperplasia associated with the use of dental prostheses in a university clinic in Medellín, 2017

\section{Margarita Rosa Jaramillo Correa ${ }^{1}$}

Recibido: 21 de febrero de 2018

Aprobado: 21 de septiembre de 2018

Publicado: 1 de julio de 2019

Cómo citar este artículo: Jaramillo Correa MR. Prevalencia de estomatitis subprotésica, queilitis angular e hiperplasia fibrosa asociadas al uso de prótesis dental en una clínica Universitaria de Medellín 2017. Rev Nac Odontol. 2019; 15(29):1-14.

doi: https://doi.org/10.16925/2357-4607.2019.02.07

Artículo de investigación. https://doi.org/10.16925/2357-4607.2019.02.07

1 Fundación Universitaria Autónoma de las Américas, Medellín, Colombia ORCID: https://orcid.org/0000-0002-4788-4400

Margarita Rosa Jaramillo Correa. Estudiante de Odontología

Correo electrónico: margarita.jaramillo@uam.edu.co 


\section{Resumen}

Introducción: una prótesis dental desadaptada puede generar diferentes lesiones en la cavidad oral, sea por el cuidado defectuoso de ésta, por inadecuada limpieza o por desajuste con respecto a los tejidos anatómicos. Dentro de las patologías que se encuentran por la utilización de prótesis dentales removibles están las estomatitis subprotésica, la queilitis angular, hiperplasia fibrosa, entre otras; estas patologías son llamadas por la OMS como lesiones de la mucosa oral.

Materiales y métodos: estudio cuantitativo de tipo transversal, con una población de 162 individuos que asistían a tratamiento en una clínica odontológica universitaria en la ciudad de Medellín, año 2017. Del total de atendidos, 53 presentaban prótesis dental removible; según criterios de selección la muestra fue de 30, aplicando muestreo no probabilístico. La recolección de los datos se realizó con fuentes primarias y secundarias, con instrumento tipo encuesta: exploración clínica e indagación de variables sociodemográficas, médicas, hábitos de vida e higiene bucal. Se realizó un análisis univariado y bivariado, con frecuencias relativas y absolutas, tablas y gráficos, procesados con software GraphPad Prism. Esta investigación se clasificó con riesgo mínimo, y con aprobación del Comité de Ética en Investigación.

Resultados y conclusiones: El 23,3\% de los pacientes presentaban estomatitis subprotésica discrimida en tipo II en un $13,3 \%$, tipo I en un $10 \%$ y ausencia de patología en el $76,7 \%$. En cuanto a la queilitis angular, un $6,6 \%$ si la presentaban y no se observó ningún caso de hiperplasia fibrosa.

Palabras clave: Stomatitis, cheilitis, queilitis angular, hyperplasia, hiperplasia fibrosa, prótesis removible.

\section{Abstract}

Introduction: A maladaptive dental prosthesis can generate different lesions in the oral cavity, either due to its defective care, due to improper cleaning or due to mismatch with respect to anatomical tissues. Among the pathologies associated with the use of removable dentures are sub-prosthetic stomatitis, angular cheilitis and fibrous hyperplasia, among others; these pathologies are defined by the WHO as lesions of the oral mucosa.

Materials and methods: A quantitative cross-sectional study was performed, with a population of 162 individuals attending treatment at a university dental clinic in the city of Medellín, 2017. Of the total patients, 53 had removable dental prosthetics; according to selection criteria, the sample was 30 , applying non-probabilistic sampling. A survey tool, using primary and secondary sources, was used for data collection; regarding clinical exploration and an investigation into the sociodemographic, medical, life habits and oral hygiene variables. A univariate and bivariate analysis was performed, with relative and absolute frequencies, tables and graphs, processed with the GraphPad Prism software. This research was classified as minimal risk, and received the approval of the Research Ethics Committee.

Results and conclusions: $23.3 \%$ of the patients presented sub-prosthetic stomatitis; $13.3 \%$ with type II, $10 \%$ with type I and an absence of the pathology for the remaining $76.7 \% .6 .6 \%$ were found to suffer from angular cheilitis and no cases of fibrous hyperplasia was observed.

Keywords: stomatitis, cheilitis, angular cheilitis, hyperplasia, fibrous hyperplasia, removable dental prosthetics.

\section{Resumo}

Introdução: Uma prótese dentária mal adaptativa pode gerar diferentes lesões na cavidade bucal, seja por seus cuidados defeituosos, por limpeza inadequada ou por descasamento de tecidos anatômicos. Entre as patologias associadas ao uso de próteses removíveis estão estomatite subprotética, queilite angular e hiperplasia fibrosa, entre outras; essas patologias são definidas pela OMS como lesões da mucosa oral.

Materiais e métodos: Foi realizado um estudo quantitativo de corte transversal, com uma população de 162 indivíduos em tratamento em uma clínica odontológica universitária na cidade de Medellín, 2017. Do total de 
pacientes, 53 possuíam próteses dentárias removíveis; de acordo com os critérios de seleção, a amostra foi de 30, aplicando amostragem não probabilística. Uma ferramenta de pesquisa, usando fontes primárias e secundárias, foi usada para coleta de dados; sobre exploração clínica e investigação das variáveis sociodemográficas, médicas, hábitos de vida e higiene bucal. Foi realizada análise univariada e bivariada, com frequências relativas e absolutas, tabelas e gráficos, processados com o software GraphPad Prism. Esta pesquisa foi classificada como risco mínimo e recebeu a aprovação do Comitê de Ética em Pesquisa.

Resultados e conclusões: $23,3 \%$ dos pacientes apresentaram estomatite subprotética; $13,3 \%$ no tipo II, $10 \%$ no tipo I e ausência da patologia nos $76,7 \%$ restantes. $6,6 \%$ apresentaram queilite angular e nenhum caso de hiperplasia fibrosa foi observado.

Palavras-chave: estomatite, queilite, queilite angular, hiperplasia, hiperplasia fibrosa, próteses dentárias removíveis.

\section{Introducción}

El concepto de salud funcional permite que el individuo viva a plenitud de acuerdo con sus posibilidades y capacidad. La salud bucal es parte esencial de la salud general y de la calidad de vida de las personas por lo que debe ser comprendida y atendida adecuadamente por los profesionales del sector (1). Dentro de los aspectos que influyen en el desarrollo del ser se encuentra el físico, por lo tanto, si un paciente pierde una pieza dentaria este busca la forma de reemplazarla ya sea por implantes, prótesis fija o prótesis removible.

La presencia de algún tipo de prótesis dental en la población colombiana se da en un 31,62 \% y este valor presenta variaciones, alcanzando el 77,43 \% de la población en edades entre 65 a 79 años (2) Es importante mencionar que la ubicación de cualquier tipo de prótesis dental en la cavidad bucal genera variaciones al interior de esta que obligan a los tejidos a adaptarse a las nuevas características del medio (3). La dentadura parcial removible (DPR) es aquella que en boca restituye funciones principales del paciente como la masticación, deglución, respiración y fonación (4). Una prótesis dental desadaptada puede generar diferentes lesiones en la cavidad oral, ya sea por el cuidado defectuoso de ésta, por la inadecuada limpieza para mantenerla libre de bacterias $u$ hongos, por el desajuste con respecto a los tejidos anatómicos o porque la boca es una cavidad en la cual hay una variación en la flora microbiana que generalmente está controlada, pero que, en caso de proliferar, puede dar origen a diversas lesiones o patologías (5).

Dentro de las patologías que se encuentran por la utilización de prótesis dental removibles están la estomatitis subprotésica, la queilitis angular, la hiperplasia fibrosa asociada a placa, entre otras (6). Según M. Rodríguez Fernández de la Universidad Autónoma de Yucatán se evaluaron lesiones en pacientes con prótesis dental de una comunidad de México; se encontró que las lesiones de mayor prevalencia en boca 
eran la estomatitis subprotésica con un 32,3\%; seguida de la hiperplasia fibrosa que fue de $14,5 \%$, la queilitis angular fue de 12,9\%, eritroplasia fue de 9,7 \% y leucoplasia con 4,8 \%; y el 24,2 \% de la muestra no presentó lesiones ni patologías clínicas en la mucosa bucal (7).

En los pacientes de edad avanzada, que son generalmente los portadores de prótesis, es conveniente implantar un programa para prevenir la aparición de dichas lesiones. En este programa preventivo se debe de incluir medidas como: profilaxis y eliminación de los residuos bacterianos en los dientes remanentes y en las prótesis en la consulta dental en intervalos máximos de seis meses (dependiendo del paciente); recambio de los cepillos dentales y los cepillos de limpieza de las prótesis; instrucciones de higiene oral y de la prótesis a pacientes y cuidadores, incluyendo el dejarla seca y no colocársela de noche y tener un correcto lavado de manos (8).

La estomatitis protésica o estomatitis subprotésica es una patología de etiología multifactorial que se manifiesta a la exploración clínica como un eritema que dibuja los límites de la prótesis (6). Existen tres tipos de estomatitis protésica, estos tipos se encuentran dentro de la Clasificación Modificada de Newton y son los siguientes: 1) inflamación simple localizada: caracterizada por la presencia de máculas eritematosas inflamatorias, localizadas alrededor de las glándulas salivales menores del paladar y petequias delimitadas en la superficie palatina. 2) inflamación simple generalizada: inflamación difusa en toda la superficie de la mucosa de soporte de la prótesis. Esta área aparece con edema, eritema brillante y puede presentar sangramiento. 3) inflamación granular o papilar hiperplásica: caracterizada porque la mucosa palatina presenta un aspecto inflamatorio eritematoso, granular o papilar (9).

Además, en el ENSAB IV realizado en Colombia registró que la estomatitis subprotésica identificada en adultos en edades de 20 a 79 años se presentaba en un porcentaje del 14,56 \% con respecto al total de población valorada para ese rango de edad (2).

De las lesiones por prótesis dental se encuentra también la queilitis angular, el cual es uno de los estados inflamatorios más comunes de las comisuras labiales en forma de úlcera. Algunos autores la clasifican dentro de las formas de presentación de la candidiasis (10). se trata de un síndrome clínico en cuya etiología se encuentran implicados varios factores en diferentes grados y combinaciones tales como (10):

1. Factores mecánicos: la desdentación y el hecho de que los pacientes sean portadores de prótesis dental mal ajustadas, ya que, la pérdida de la dimensión vertical produce pliegues comisurales, cuya humidificación por la saliva favorece la maceración angular (11). 
2. Factores infecciosos: es frecuente la sobreinfección de las comisuras por gérmenes, tales como Candida albicans y Staphylococcus aureus, si bien se han descrito también infecciones comisurales por Streptococcus haernolyticus y Streptococcus agalactiae (11).

3. Trastornos carenciales: existen una serie de situaciones carenciales en la dieta que aumentan la vulnerabilidad de la mucosa labial y, por tanto, predisponen a la queilitis angular. Los estados carenciales de hierro y las anemias ferropénicas producen también cambios mucosos que incluyen queilitis angular, glositis y atrofia de las papilas filiformes de la lengua. La deficiencia de hierro parece facilitar algunas infecciones, especialmente las debidas a Candida albicans (11).

4. Trastornos cutáneos: existen una serie de trastornos cutáneos que condicionan una mayor sequedad de piel y mucosas, como es el síndrome de Sjogren y las dermatitis atópicas (11).

Clínicamente la queilitis angular se presenta como una zona triangular de eritema y edema en una o ambas comisuras de la boca. Se observa un proceso inflamatorio, que posteriormente se fisura y produce exudación y costras. De aquí la lesión se extiende hacia la piel. La mucosa vecina no se afecta, salvo en casos de candidiasis oral generalizada (11)

Otra de las lesiones es la hiperplasia fibrosa asociada a prótesis; ésta es una lesión reactiva de la mucosa oral generalmente asociada a prótesis mal adaptadas (12) debido a que se genera una disminución del soporte (por la reabsorción alveolar) y la prótesis dental se profundiza poco a poco sobre el fondo de surco, ejerciendo una presión anormal sobre los tejidos blandos. Aquí se combinan dos lesiones: úlcera crónica e hiperplasia de tejido conjuntivo crónicamente inflamado (13). En las primeras etapas el irritante crónico estimula la formación de tejido de granulación; más adelante, el tejido comienza a sufrir un proceso de fibrosis. La presencia de factores irritativos en la mucosa desencadena un proceso inflamatorio crónico que culmina con la formación de tejido fibroso hiperplásico asintomático, en las zonas de agresión. (13)

Un estudio de Anita Rodríguez y Sonia Sacsaquispe encontraron que en los adultos mayores (de más de 60 años) portadores de prótesis dental removible, la prevalencia de la hiperplasia fibrosa inflamatoria asociada al uso de prótesis dental removible y sus factores asociados fue del 26,4 \% (91 de los sujetos examinados) (14). Además, se reportó también a partir de éste la importancia de evaluar el compromiso sistémico de cada uno de los sujetos, pues al compararlos con el desarrollo de la $\mathrm{HFI}$, encontraron una mayor frecuencia de esta lesión en sujetos sistémicamente 
comprometidos (27,6\%). Las principales enfermedades encontradas fueron: hipertensión arterial, Parkinson, diabetes, problemas cardíacos y otros (hipercolesterolemia, cefaleas, artritis, artrosis), aunque todos estaban controlados (14).

El objetivo de esta investigación fue identificar la prevalencia de estomatitis subprotésica, queilitis angular e hiperplasia fibrosa asociada a prótesis dental en la Clínica Odontológica Fundación Universitaria Autónoma de las Américas, tomando como referencia de estudio pacientes usuarios de este servicio.

\section{Materiales y métodos}

Se realizó un estudio de enfoque cuantitativo descriptivo de tipo transversal; la población seleccionada estaba integraba por 162 individuos que asistían a consulta odontológica en una asignatura de Atención Integral del Adulto en una clínica de formación del pregrado de odontología; éste fue desarrollado en el año 2017. Del total de la población, 53 de los sujetos para el estudio fueron seleccionados mediante un muestreo no probabilístico a conveniencia, teniendo en cuenta los criterios de inclusión como: pacientes que asistieran a la asignatura de Atención Clínica VII de adulto, con historia clínica diligenciada completamente en la institución, que presentaran condiciones de edentulismo y poseedores de aparatología removible (total, removible, estética e interina), estar de acuerdo en la participación y firmar el consentimiento informado. Dentro de los criterios de excusión están pacientes menores de 30 años o que presentaran aparatología fija; no se excluyeron los pacientes con patologías sistémicas ya que estas pueden influir en la aparición de hiperplasia fibrosa asociada a prótesis.

La recolección de los datos se realizó por medio de dos fuentes de información las cuales tenían como instrumento una encuesta. El instrumento de recolección no ha sido validado, sino que fue desarrollado dentro del proyecto, así que es de carácter original. En la fuente primaria se realizó una exploración clínica y una encuesta por medio de la cual se recopilaros las siguientes variables: sociodemográficas como edad, sexo, estrato socioeconómico, y nivel de escolaridad. Contó además con variables clínicas como: antecedentes médicos, hábitos de vida, hábitos de higiene bucal, examen bucal para identificar presencia de lesiones intrabucales y extrabucales y la descripción de estas. En la fuente secundaria se evaluaron variables tales como: edad, sexo, hallazgos de lesiones en ronda estomatológica, registro de lesiones en diagnóstico y plan tratamiento, extrayendo esta información de la historia clínica personal de 
cada individuo participante de la investigación, es importante aclarar que la historia clínica debía estar completamente diligenciada.

La recolección de los datos se realizó dentro de la clínica de la Universidad, cumpliendo todas las medidas de bioseguridad requeridas; además la estudiante que realizó los exámenes clínicos fue calibrada y orientada en el trabajo de campo por una docente odontóloga y especialista en patología oral, quien estuvo presente en la recolección de datos para darle confiabilidad a los mismos. El proceso de recolección se realizó primero explicando el proyecto y el consentimiento informado los participantes para su posterior firma, luego el diligenciamiento de la encuesta y la exploración clínica del paciente. Dicha exploración se realizó con la luz de las unidades de la clínica y un espejo bucal. Se valora adaptación de la prótesis y al encontrar estas en condición de desadaptación, se procedía a removerla para poder evaluar si presentaba alguna de las lesiones. Posteriormente se pasó a revisar cada una de las historias clínicas de los pacientes, teniendo la previa autorización de los pacientes. El procesamiento y análisis de datos fue realizado con el software GraphPad Prism con Licencia Fundación Universitaria Autónoma de las Américas. Se realizó un análisis univariado para obtener frecuencias relativas y absolutas, tablas de frecuencia y gráficos dinámicos, además de análisis bivariado cruzando una variable dependiente con varias independientes, acorde a los resultados de la descriptiva inicial haciendo prueba de hipótesis para Chi cuadrado para observar diferencias estadísticamente significativas entre las variables del estudio.

Se contó con la aprobación del Comité de Ética en Investigación de la Fundación Universitaria Autónoma de las Américas, dando garantía al respeto por la dignidad humana, la confidencialidad y el anonimato. Se contó con consentimiento informado y la declaración de adherencia a todos los protocolos internacionales. Esta investigación fue clasificada con riesgo mínimo.

\section{Resultados}

La muestra de este estudio estuvo conformada por 30 sujetos los cuales cumplieron con los criterios de inclusión. Con la ejecución de este proyecto se obtuvo información sobre la prevalencia de patologías asociadas a prótesis removibles dentales sea total, parcial, interina o inmediata como son la estomatitis subprotésica, queilitis angular e hiperplasia fibrosa.

Según los datos obtenidos en el estudio se observó ausencia total de la patología hiperplasia fibrosa que era una de las lesiones a evaluar. La prótesis que se 
presentaba con mayor frecuencia en los pacientes evaluados fue la prótesis parcial removible, en un $40 \%$.

Es importante señalar, acorde a la tabla 1, que el 46,7 \% de los encuestados tenían prótesis en boca entre 1 y 10 años o más.

Tabla 1. Tiempo con la prótesis dental.

\begin{tabular}{llll}
\hline & & N & \multicolumn{1}{c}{$\%$} \\
\hline MENOS DE 6 MESES & 10 & 33,3 \\
\hline ENTRE 6 MESES Y 1 AÑO & 6 & 20,0 \\
\hline ENTRE 1 AÑO Y 5 AÑOS & 6 & 20,0 \\
\hline ENTRE 5 A 10 AÑOS & 5 & 16,7 \\
\hline MAS DE 10 AÑOS & 3 & 10,0 \\
\hline Total & 30 & 100,0 \\
\hline
\end{tabular}

Fuente: elaboración propia

Al evaluar los pacientes con estomatitis protésica se clasificó según la gradificación de Newton (estomatitis subprotésica tipo I: inflamación simple localizada, tipo II: inflamación simple generalizada, y tipo III: inflamación granular o papilar hiperplásica) y se encontró que 23 pacientes (76,7 \%) no presentan dicha patología;estomatitis subprotésica tipo II 4 pacientes (13,3\%), la tipo I 3 pacientes (10\%) yy la tipo III no se presento (tabla 2).

Tabla 2. Prevalencia de estomatitis subprotesica.

\begin{tabular}{llll}
\hline & & $\mathbf{N}$ & $\%$ \\
\hline TIPO I & 3 & 10,0 \\
\cline { 2 - 4 } TIPO II & 4 & 13,3 \\
\cline { 2 - 4 } NO LA PRESENTA & 23 & 76,7 \\
\cline { 2 - 4 } & Total & 30 & 100,0 \\
\hline
\end{tabular}

Fuente: elaboración propia

En cuanto a la queilitis angular asociada a prótesis dental se encontró que 28 pacientes $(93,3 \%)$ no presentan dicha patología y que 2 pacientes (14,3\% ) si la presentaban (tabla 3). 
Tabla 3. Prevalencia de queilitis angular asociada a prótesis.

\begin{tabular}{llll}
\hline & $N$ & $\%$ \\
\hline NO LA PRESENTA & 28 & 93,3 \\
\cline { 2 - 3 } SI LAPRESENTA & 2 & 6,7 \\
\hline Total & 30 & 100,0 \\
\hline
\end{tabular}

Fuente: elaboración propia

En cuanto a los datos recolectados en las historias clínicas de los pacientes evaluados en esta investigacion se encontró que los estudiantes reportan alguna de las patológías observadas clínicamente en un 13,3 \% y no aparece reportado eldiagnóstico de estas patologías en un 13,3\% (tabla 4).

Tabla 4. Diagnósticos de las patologías estomatitis, queilitis e hiperplasia asociada a prótesis en la historia de la UAM.

\begin{tabular}{|c|c|c|}
\hline & $\mathrm{N}$ & $\%$ \\
\hline NO DEFINEN LA PATOLOGÍA & 4 & 13,3 \\
\hline OCLUSAL & 4 & 13,3 \\
\hline SANO & 22 & 73,3 \\
\hline Total & 30 & 100,0 \\
\hline
\end{tabular}

Fuente: elaboración propia

Con respecto a los paciente que presentaron lesiones, se observó que los estudiantes no definen el plan de tratamiento para dichas patologías en 6 historias clínicas (20\%) y lo definen en solo 2 historias (6,7 \%) (tabla 5).

Tabla 5. Plan de tratamiento de las patologías estomatitis, queilitis e hiperplasia asociada a prótesis en la historia clínica de la FUAM.

\begin{tabular}{llll}
\hline & & $\mathbf{N}$ & $\%$ \\
\hline ANTIINFECCIOSO & 2 & 6,7 \\
\cline { 2 - 4 } NO DEFINEN LA PATOLOGÍA & 6 & 20,0 \\
\cline { 2 - 4 } SANO & 22 & 73,3 \\
\hline Total & 30 & 100,0 \\
\hline
\end{tabular}

Fuente: elaboración propia

Se observó que la estomatitis subprótesica tipo II en mujeres fue más prevalente con 3 pacientes (75 \%) en comparación con los hombres que fue 1 solo (25 \%);la tipo I se 
presentó en hombressolo 1 vez que equivale al 33,3 \% y mujeres 2 veces correspondiendo a un $66,7 \%$. Además,fuemayor el porcentaje de mujeres que no presentaban dicha patología (65,2 \%) en comparación con los hombres (34,8 \%) (figura 1).

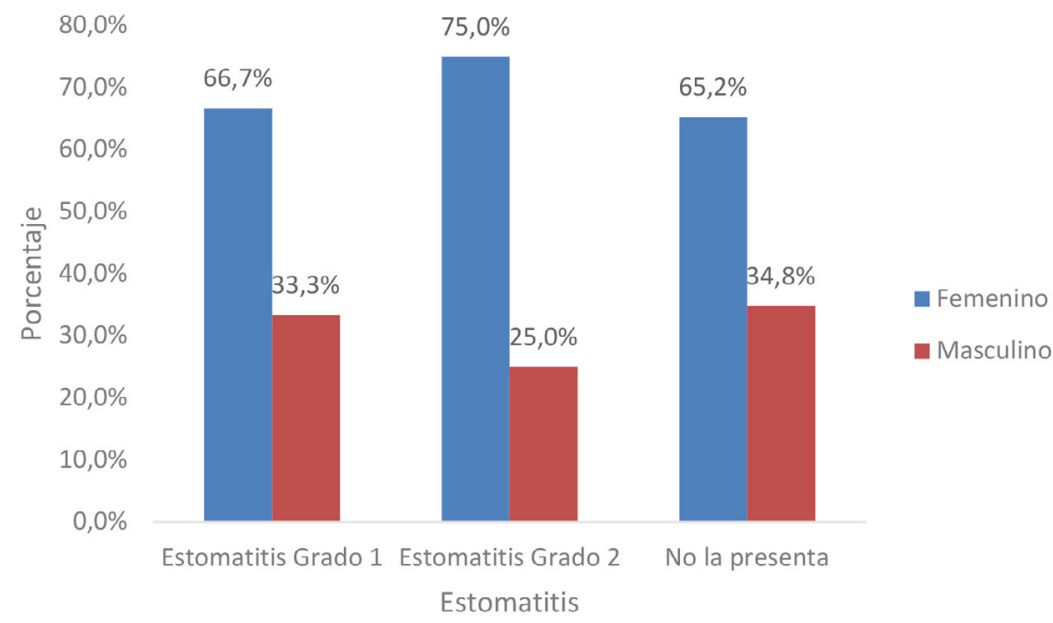

Figura 1. Distribución porcentual de prevalencia de estomatitis subprotésica según sexo. Fuente: elaboración propia

La patología de queilitis angular asociada a la prótesis solo se encuentró en dos mujeres de todos los pacientes evaluados. Y los que no presentaron dicha lesión mostraron una distribucion de $64,3 \%$ en mujeres y $35,7 \%$ en hombres (figura 2).

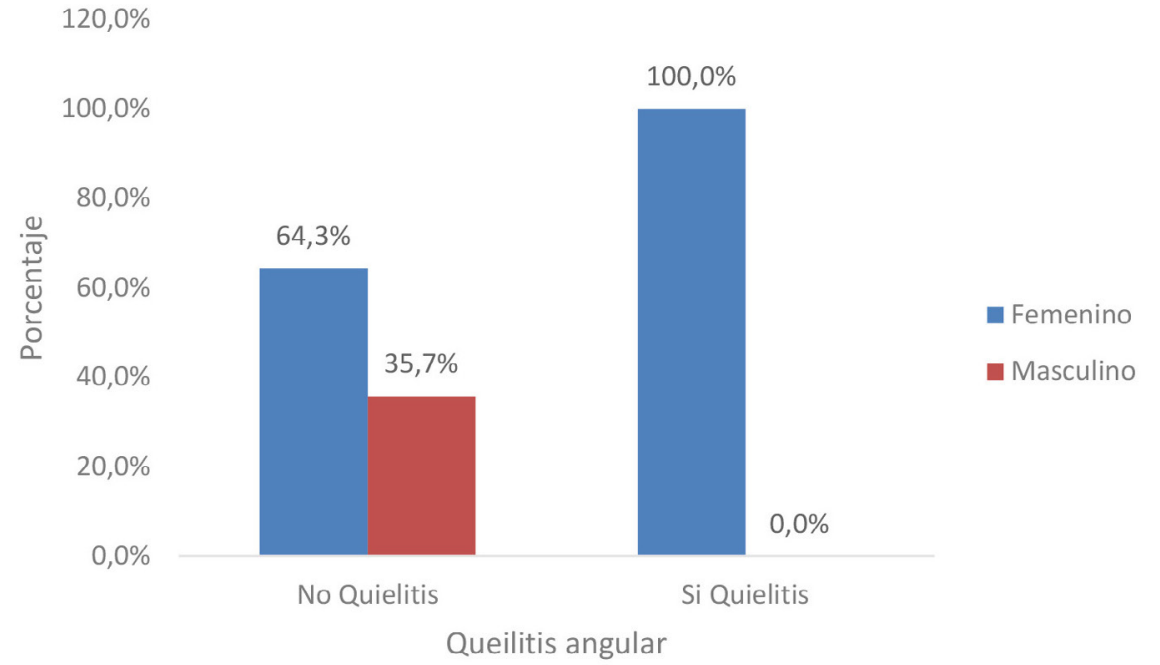

Figura 2. Distribución porcentual de prevalencia de queilitis angular asociada a prótesis según sexo.

Fuente: elaboración propia 
Se observó que los pacientes menores de 52 años presentaban estomatitis subprotésica tipo 2 en un 50 \%; en el rango de edad entre 53 a 62 y entre 63 a 72 años se encontró esta misma patología en el mismo porcentaje (25\%). En cuanto a la estomatitis subprotésica tipo 1, en el rango de edad entre 53 y 62 años, fue del 33,3 \% al igual que en los rangos de 63 a 72 años y de 73 años en adelante (figura 3).

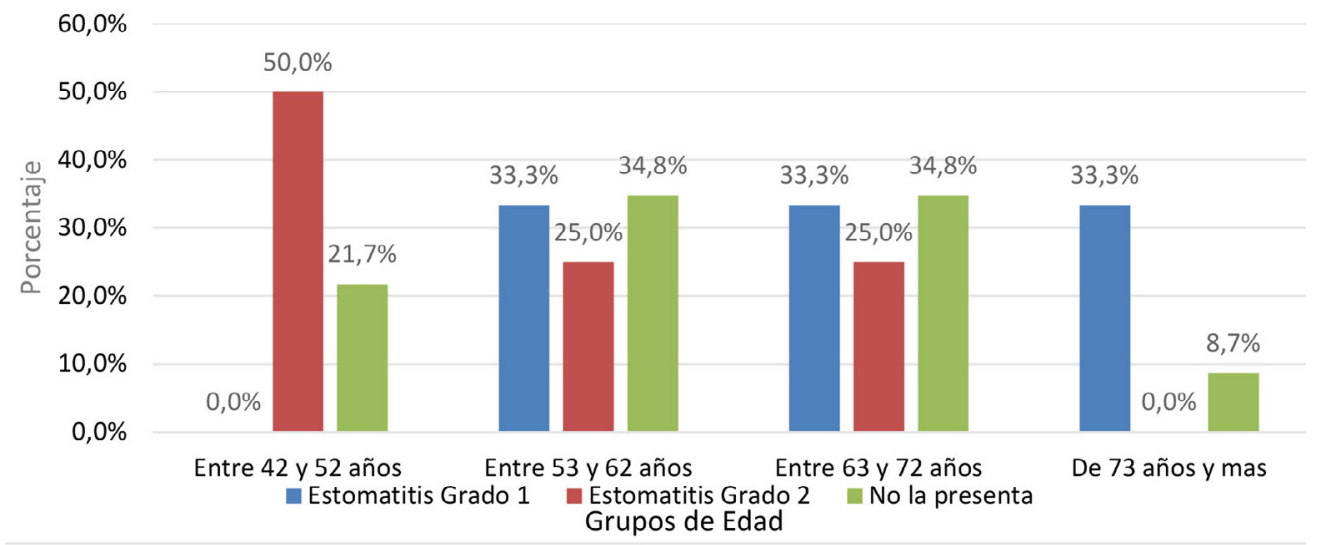

Figura 3. Distribución porcentual de prevalencia de estomatitis subprotésica según grupos de edad.

Fuente: elaboración propia

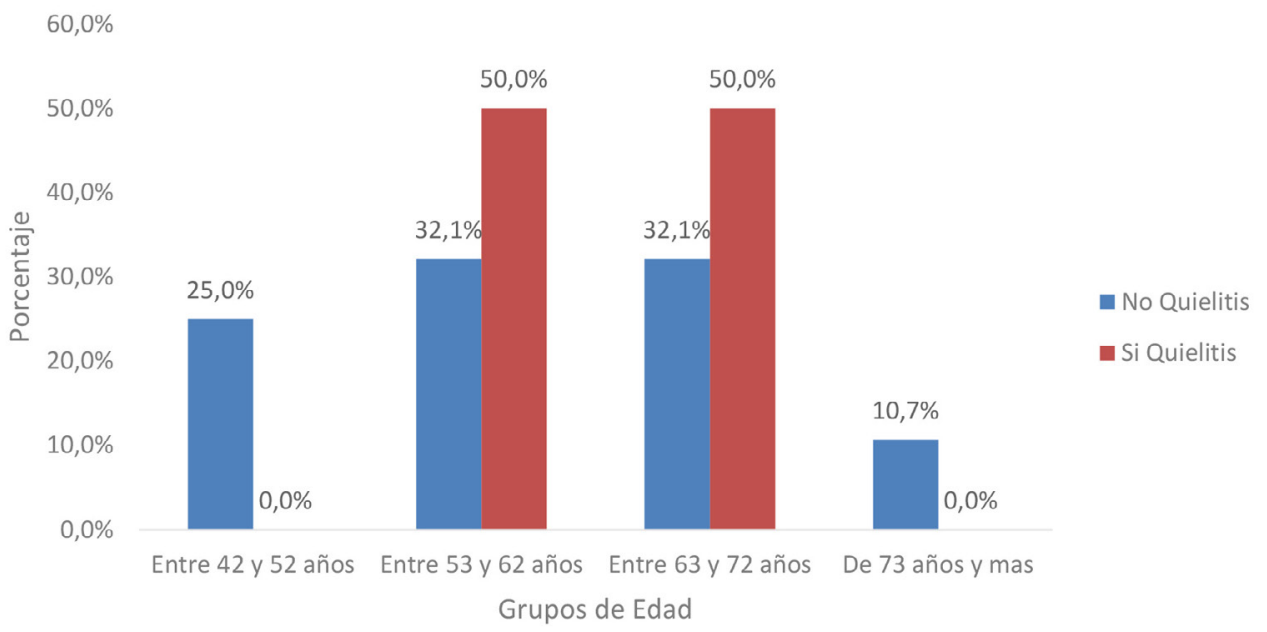

Figura 4. Distribución porcentual de prevalencia de Queilitis angular asociada a prótesis según grupos de edad.

Fuente: elaboración propia 
En cuanto a los pacientes que presentaron queilitis angular se observó que el 50 \% estaban en un rango de edad entre los 53 y 62 años y en el mismo porcentaje en aquellos que estaban en el rango de edad entre 63 y 72 años, equivalente una persona en cada rango (figura 4).

Entre las patologías que evaluaron en esta investigación, la de mayor prevalencia fue la estomatitis asociada a prótesis dental y la de menor fue la hiperplasia fibrosa, por ausencia total.Otro resultado significativo fue que los estudiantes de la clínica del noveno y decimo semestre 2017 de la Fundación Universitaria Autónoma de las Américas en gran porcentaje no reportan las patologías.

Al cruzar la variable dependiente sexo con estomatitis, queilitis angular e hiperplasia fibrosa no se presentaron diferencias estadísticamente significativas $(p<0,05)$.

\section{Conclusiones}

Al identificar la prevalencia de estomatitis protésica, queilitis angular e hiperplasia fibrosa asociada a prótesis dental en la Clínica Universitaria, en la asignatura de Atención Clínica Integral VI (clínica del adulto) 2017, se encontró que la patología de hiperplasia fibrosa no la presentaba ninguno de los pacientes evaluados, y que la de mayor prevalencia fue la estomatitis protésica.

Los pacientes evaluados que presentaron la patología de queilitis angular no son de mucha relevancia en comparación de los que no la presentaron, porque la diferencia porcentual es muy grande. En cambio, la estomatitis subprotésica se encontró de dos de las tres formas en que se presenta, que fue el tipo I y el tipo II, de las cuales primó esta última. Con estos resultados se puede llegar a la conclusión que, la patología de mayor prevalencia es la estomatitis protésica, seguida de la queilitis angular en los pacientes de esa Clínica, según esta investigación.

La realización de esta investigación en la Universidad, se enfocó en patologías asociadas a prótesis dental, lo que permitió encontrar que el tratamiento para las patologías estomatitis protésica, hiperplasia fibrosa y queilitis angular no se realiza o no están reportadas en las historias clínicas al igual que se omite el diligenciamiento de estas en los aspectos de diagnóstico.

Al obtener los resultados de esta investigación es importante incentivar el correcto diligenciamiento de la historia clínica, ya que ésta es la fuente de información que facilita el conocimiento del progreso del paciente en su estado de salud tanto general como odontológico; además permite que todo personal de la salud pueda tener la información para saber tratar al paciente en caso de un cambio en el personal clínico. 


\section{Referencias}

1. Joya LD, Quintero LE. Caracterización de la salud bucal de las personas mayores de 65 años de la ciudad de Manizales, 2008. Hacia Promoc. Salud. 2015; 20(1): 140-152. doi: 10.17151/ hpsal.2015.20.1.10,

2. Ministerio de Salud. IV Estudio Nacional De Salud Bucal [Internet]. Colombia: Ministerio de Salud; [consulta 03/09/2016]. Disponible en: https://www.minsalud.gov.co/sites/rid/Lists/ BibliotecaDigital/RIDE/VS/PP/ENSAB-IV-Situacion-Bucal-Actual.pdf

3. Felipe RN, Delgado JA, Soto G. Factores de Riesgo en las lesiones premalignas del complejo bucal. Rev Cubana Estomatol.1995; 32(2): 1-4.

4. Velazquez A, Florentín GD, Defazio D. Frecuencia de estomatitis subprotésica en pacientes portadores de prótesis dentales removibles. Rev Fac Cienc Salud UDES. 2017; 4(1): 45-50. doi: http://dx.doi.org/10.20320/rfcsudes.v4i1.106

5. Hidalgo-Hidalgo S, Ramos-Díaz M. Alteraciones bucales en pacientes geriátricos portadores de prótesis total en dos áreas de salud [Internet]; [consulta 28/09/2016]. Disponible en: http://scielo.sld.cu/pdf/amc/v9n6/amc010605.pdf

6. Barata-Caballero D, Durán-Porto A, Carrillo-Baracaldo S. Estomatitis protésica. Aspectos clínicos y tratamiento. Prof Dent. 2002; 5(10): 622-27.

7. Rodríguez-Fernández MDSC, Portillo-Recio A. Lesiones bucales asociadas con el uso de prótesis en pacientes de la comunidad de Kantunil, Yucatán. Revista ADM. 2014; 71(5): 221-225.

8. Ayuso-Montero. R, Torrent-Collado J, López-López J. Estomatitis protésica: puesta al día. RCOE. 2004; 9(6): 657-662. doi: 10.4321/S1138-123X2004000600004\}

9. Corzo M, Diana Annabella. Estomatitis protésica o por dentadura. Universidad de San Carlos de Guatemala. Facultad de Odontología. Área de patología [Internet]; [consulta: 26/09/16]. Disponible en: https://4tousac.files.wordpress.com/2012/01/estomatitis-protc3a9sicas.pdf

10. García LópezE, Blanco RuizA. Queilitis. Revisión bibliográfica [Internet]; [consulta 03/09/2016]. Disponible en: http://www.bvs.sld.cu/revistas/est/vol41_2_04/est09204.htm

11. Chimenos Küstner E. Queilitis angular malignizada a propósito de un caso. Avances en Odontoestomatología. 1992; 8: 37-41. 
14 Prevalencia de estomatitis subprotésica, queilitis angular e hiperplasia fibrosa asociadas al uso de prótesis dental en una clínica universitaria de Medellín, 2017

12. Vargas PA, Pérez DEC, Jorge J, Rangel ALCA, León JE, Almeida OE. Denture hyperplasia with areas simulating oral inverted ductal papilloma. Med Oral Patol Oral Cir Bucal. 2005; 10 Suppl2: E117-21.

13. Casian Romero, Trejo Quiroz, De León Torre, Carmona Ruiz. Hiperplasia Fibrosa Inflamatoria: reporte de un caso. Rev Clin Periodoncia Implantol Rehabil Oral. 2011; 4(2): 74-79.

14. Rodríguez A, Sacsaquispe S. Hiperplasia fibrosa inflamatoria y posibles factores asociados en adultos mayores. Rev Estomatol Herediana. 2005; 15(2): 139-144. 